\title{
Response to Trastuzumab and Lapatinib in a Metastatic Colorectal Cancer Harboring HER2 Amplification and HER2 S310F Mutation
}

\author{
Chongkai Wang, MD, MS, ${ }^{1}$ and Marwan Fakih, $\mathrm{MD}^{1}$
}

\begin{abstract}
Dual HER2-targeted therapy has been associated with clinical responses and prolonged progression-free survival and overall survival in RAS-wild type HER2-amplified colorectal cancer (CRC). However, no clinical benefits have been reported in patients with CRC with HER2 mutations. Activated HER2 mutations have been largely deemed resistant to trastuzumab and to dual HER2 targeting. This report describes a patient with metastatic CRC with concurrent HER2 amplification and a HER2 S310F mutation, which is an active mutation located in the extracellular dimerization domain of HER2. Treatment with trastuzumab + lapatinib resulted in an excellent response that lasted for 10 months. Upon disease progression, treatment with the antibody-drug conjugate trastuzumab-deruxtecan resulted in a short-lived response. This is the first case report of successful HER2 targeting in metastatic CRC with concurrent HER2 amplification and a HER2 S310F mutation.
\end{abstract}

J Nat/ Compr Canc Netw 2021;19(6):670-674 doi: 10.6004/jnccn.2021.7023

${ }^{1}$ Department of Medical Oncology and Therapeutics Research, City of Hope Comprehensive Cancer Center, Duarte, California.
HER2 amplification occurs in $2 \%$ to $3 \%$ of patients with metastatic colorectal cancer (CRC). ${ }^{1-3}$ Recent studies have shown that HER2 amplification is associated with relative resistance to anti-epidermal growth factor receptor (EGFR) targeting in RAS wild-type (WT) CRC. ${ }^{4-6}$ Preclinical studies have conferred the sensitivity of HER2-amplified RAS WT colorectal patient-derived xenograft (PDX) models to dual HER2 targeting, leading to several phase II clinical trials in patients with RAS WT metastatic CRC. ${ }^{7}$ The combinations of trastuzumab + lapatinib, trastuzumab + pertuzumab, and trastuzumabderuxtecan have all been associated with significant activity in HER2-amplified RAS WT CRC, leading to their endorsement in the NCCN Clinical Practice Guidelines in Oncology (NCCN Guidelines) for Colon Cancer as a treatment option for this patient population. ${ }^{8-11}$

HER2 mutations are equally rare in metastatic CRC, occurring in approximately $2 \%$ of patients. ${ }^{12}$ For example, concurrent HER2 amplification and mutation was found in $0.5 \%$ of patients with CRC. ${ }^{12}$ Preclinical studies have shown that select activated HER2 mutations are sensitive to the combination of the HER2 antibody + HER2 tyrosine kinase inhibitors (TKIs). ${ }^{13}$ Responses to HER2targeted therapy have been reported in HER2-mutated breast cancer. ${ }^{14}$ However, no convincing clinical activity has been reported yet in metastatic CRC. A comprehensive investigation of HER2 alterations in metastatic CRC showed that HER2 mutations were clustered in the tyrosine kinase domain, the juxtamembrane domain, the transmembrane domain, and the extracellular domain (ECD) ${ }^{12}$ Mutations in the tyrosine kinase domain, such as L755S, V842I, D769Y, and K753E, lead to increased kinase activity and have been associated with resistance to both anti-HER2 antibodies and small-molecule HER2 kinase inhibitors. ${ }^{15-18}$ Responses to HER2 targeting have been reported in juxtamembrane domain/transmembrane domain mutations in preclinical and clinical studies. ${ }^{19,20}$ Mutations in the ECD, such as S310F and S310Y, lead to the increased dimerization of HER2 and subsequent activation of the downstream signaling pathways. Prior 
studies have shown that these ECD mutations do not affect trastuzumab binding. Cells overexpressing these mutated proteins remain sensitive to both trastuzumab and HER2 TKIs, such as lapatinib, neratinib, and afatinib. ${ }^{21,22}$ Given the unique mechanism of activation of S310F HER2 mutations, it is more likely that these mutations may be amenable to inhibition in metastatic CRC. This report describes the first patient with $R A S$ WT metastatic CRC and a concurrent HER2 amplification and HER2 S310F mutation who experienced a clinical response to dual HER2 targeting.

\section{Case Presentation}

A male patient aged 59 years presented with rectal bleeding, leading to diagnosis of a clinically T3N0 poorly differentiated rectal adenocarcinoma in November 2016. He received capecitabine-based chemoradiation between November 2016 and January 2017 and experienced a complete clinical response, after which he opted for watchful waiting and no further treatment. In June 2017, he was found to have disease recurrence with distant lymph node metastases. Biopsy of a supraclavicular lymph node revealed a RAS and BRAFWT microsatellitestable metastatic adenocarcinoma, consistent with his primary tumor. He was treated with infused 5-FU/folinic acid/oxaliplatin (FOLFOX) + bevacizumab between July 2017 and November 2017, with an excellent response, followed by further consolidation radiation therapy to the supraclavicular lymph node area. The patient experienced further disease progression in May 2018 with confirmed metastatic disease to the liver; he was started on 5-FU/folinic acid/irinotecan (FOLFIRI) + panitumumab and completed 6 cycles of treatment. Imaging studies showed response to treatment, and radiofrequency ablation of 3 hepatic lesions was performed in late August 2018. In November 2018, he was diagnosed with bowel obstruction secondary to progressive local recurrence that required a diverting ileostomy. Intraoperative findings were significant for the presence of peritoneal carcinomatosis, and this was confirmed through biopsy. The patient was referred to our center at that point for consideration for clinical trials.

A circulating tumor DNA (ctDNA) assay was performed (Guardant360; Guardant Health, Inc.) to explore the presence of other potential driver mutations or potential mechanisms of resistance to anti-EGFR therapy. Results showed a plasma HER2 copy number of 8.1 and a HER2 S310F mutation (variant allele frequency [VAF], $72.3 \%$ ) in addition to TP53 S127fs and MYC amplification. Given the recently documented predictive value of the HER2 ctDNA copy number and response to trastuzumab + lapatinib, and knowing the potential sensitivity of S310F to HER2 targeting, the patient was offered prompt treatment with dual HER2 targeting. ${ }^{23}$ In December 2018, he was started on trastuzumab at an intravenous loading dose of $8 \mathrm{mg} / \mathrm{kg}$, followed by $6 \mathrm{mg} / \mathrm{kg}$ intravenously every 3 weeks plus oral lapatinib at 1,000 mg once daily.

Within 2 months, the patient's performance status dramatically improved from ECOG 2 to 0 , along with a significant decline in tumor markers (Figure 1A). Significant tumor shrinkage was documented on his imaging studies (Figure 1B, C) after 8 cycles of treatment. He continued this treatment for 13 cycles with an excellent response, but experienced disease progression in August 2019. Repeat Guardant360 ctDNA assay at the time of progression revealed an increased plasma copy number of HER2 amplification (14.8), increased allele frequency of the HER2 S310F mutation (VAF, 85.6\%), HER2 G1189fs mutation (variant of uncertain significance), and FGFR2 amplification (plasma copy number 3.0), in addition to KRAS G12V, PIK3CA R88Q, PIK3CA E545K, and TP53 S127fs mutations and MYC amplification (Figure 2A, B). A liver biopsy confirmed ongoing HER2 amplification and the presence of the HER2 S310F mutation, in addition to a PIK3R1 E17fs mutation, FANCD2 mutation, and MYC amplification in late September 2019. The patient was rechallenged with FOLFOX + bevacizumab (September 2019 to March 2020), but experienced disease progression in the liver, distant lymph node, and primary tumor. He then received 3 cycles of trastuzumab + pertuzumab
A

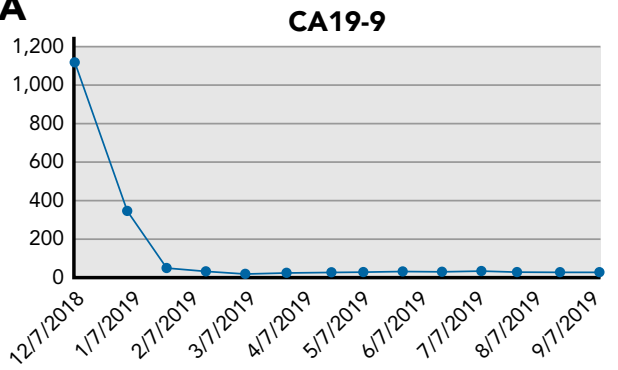

B

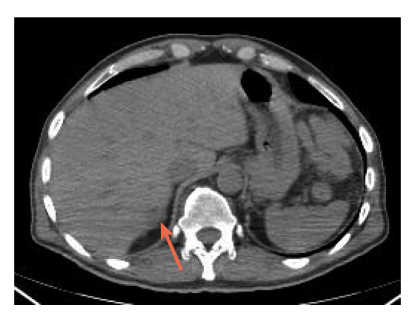

C

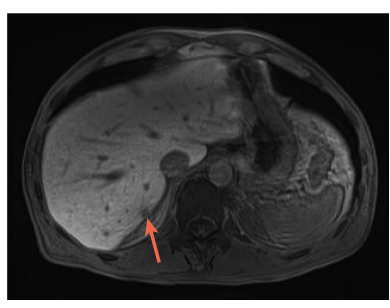

Figure 1. Laboratory and imaging results after treatment with trastuzumab + lapatinib. (A) Graph showing the dynamics of CA19-9 tumor marker response during treatment. (B) CT image from PET/CT scan before trastuzumab + lapatinib (red arrow: liver lesion, $2.4 \times 1.4 \mathrm{~cm}$ ). (C) MRI scan after 8 cycles of trastuzumab + lapatinib (red arrow: the same lesion, $0.7 \times 0.9 \mathrm{~cm}$ ). 
A

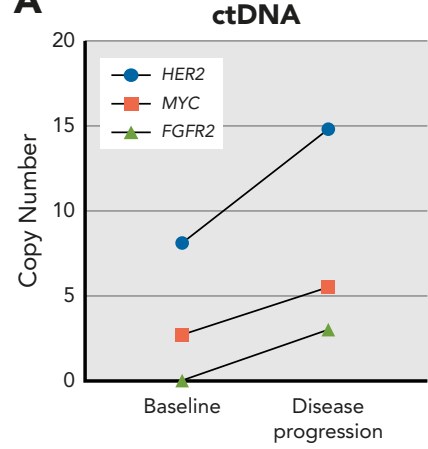

B

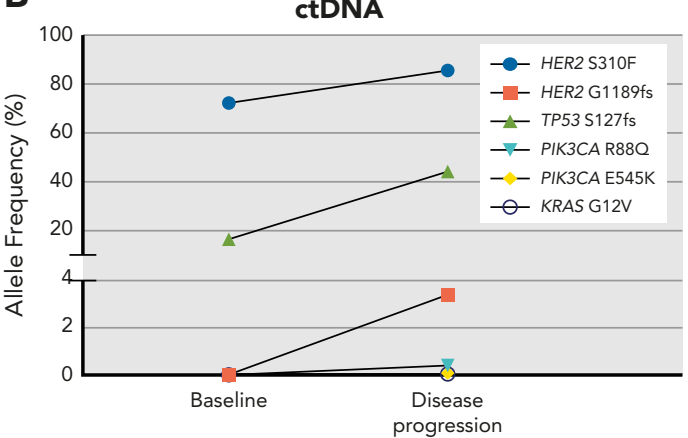

Figure 2. ctDNA change after trastuzumab and lapatinib treatment. (A) Plasma copy number change of HER2, MYC, and FGFR2: copy numbers of HER2, MYC, and FGFR2 at baseline were 8.1,2.7, and 0, respectively, and the time of disease progression were 14.8, 5.5, and 3.0, respectively. (B) VAF change in HER2, TP53, PIK3CA, and KRAS: VAFs of the HER2 S310F, HER2 G1189fs, TP53 S127fs, PIK3CA R88Q, PIK3CA E545K, and KRAS G12V mutations at baseline were $72.3 \%, 0 \%, 16.5 \%, 0 \%, 0 \%$, and $0 \%$, respectively, and at the time of disease progression were $85.6 \%, 3.4 \%, 44.2 \%, 0.4 \%, 0.08 \%$, and $0.04 \%$, respectively.

Abbreviations: ctDNA, circulating tumor DNA; VAF, variant allele frequency.

between April 2020 and June 2020, with continued disease progression. Given the reported activity with trastuzumab-deruxtecan in the DESTINY-CRC01 trial in patients with prior trastuzumab-naïve and pretreated metastatic HER2-amplified metastatic $\mathrm{CRC},{ }^{11}$ the patient was treated with trastuzumab-deruxtecan from June 2020 through October 2020, and experienced a brief response in tumor markers (Figure $3 \mathrm{~A}$ ) and with tumor shrinkage in measurable lesions (Figure 3B, C), followed by disease progression.

\section{Discussion}

This case report presents the first patient with CRC with concurrent HER2 amplification and an HER2 S310F mutation who experienced remarkable response to trastuzumab + lapatinib. After 10 months, his disease progressed and treatment with trastuzumab-deruxtecan produced a short-lived response.

Dual HER2 targeting with trastuzumab + HER2specific TKIs (lapatinib or tucatinib) or trastuzumab + pertuzumab has achieved significant clinical activity in multiple phase II trials in RAS/RAF WT, HER2-amplified CRC. ${ }^{8,9,24,25}$ Targeting HER2 mutations in CRC is not welldefined. Prior studies evaluating HER2 mutations in breast cancer and non-small cell lung cancer have shown that most of the activated HER2 mutations fall into residues in the ECD and the kinase domain. ${ }^{21,22}$ The HER2 S310F mutation in our patient was an activated mutation located in the extracellular dimerization domain and did not affect trastuzumab binding. It resulted in the noncovalent dimerization of HER2 and subsequent activation of downstream signaling. ${ }^{21}$ Preclinical studies with CRC PDX harboring an HER2 S310Y mutation, which functions the same way as the S310F mutation, showed that dual HER2-targeted therapy with trastuzumab + lapatinib leads to significant tumor regression. ${ }^{13}$ Responses to single HER2 tyrosine kinase targeting have been reported in HER2-mutated breast cancer. ${ }^{14}$ The 10-month time to progression and objective radiographic response documented in our patient confirms the clinical relevance of targeting this mutation in the setting of HER2 amplification with trastuzumab + lapatinib in patients with RAS WT metastatic CRC.
A

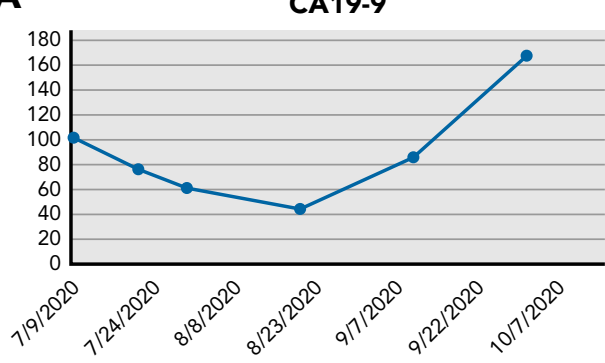

B

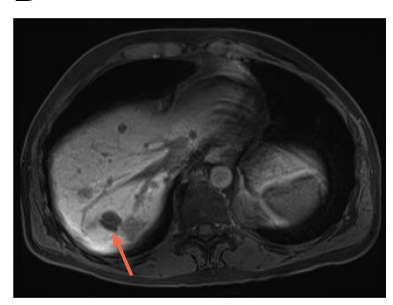

C

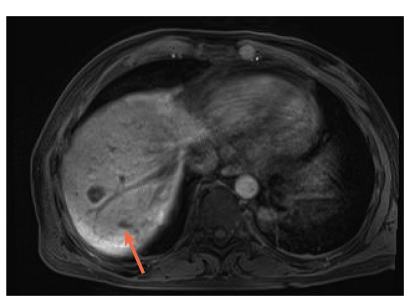

Figure 3. Laboratory and imaging results with trastuzumab-deruxtecan treatment. (A) Graph showing the dynamics of CA19-9 tumor marker response during treatment. (B) MRI scan before trastuzumab-deruxtecan therapy (red arrow: posterior hepatic dome lesion, $2.0 \times 1.9 \mathrm{~cm})$. (C) MRI scan after 4 cycles of trastuzumab-deruxtecan treatment (red arrow: the same lesion, $1.3 \times 0.7 \mathrm{~cm}$ ). 
ctDNA HER2 amplification is a reliable alternative to tissue-based genomic analysis, with an observed plasma copy number of $>2.4$ predicting response to HER2-targeted therapy. ${ }^{23}$ Indeed, our patient had a HER2 plasma copy number of 8.1 and responded to dual HER2 targeting. The genomic analysis of the liver biopsy was concordant with ctDNA assay at the time of disease progression in detecting both HER2 amplification and HER2 mutation. However, ctDNA analysis detected more genomic alterations than tumor biopsy genomic analysis, showing the limitation of tumor biopsies in mapping out genomic heterogeneity. The increase in the HER2 copy number and mutant allele frequency of the HER2 S310F mutation at the time of disease progression probably reflects the increased tumor burden at the time of progression. A novel clone of HER2 (G1189; VAF, 3.4\%), a truncate mutation localized at the distal portion of the C-terminal tail, was noticed at the time of disease progression. We did not find any reports correlating this specific mutation to HER2 resistance, and several studies have suggested that deletions in this region are not likely to affect HER2 phosphorylation. ${ }^{26,27}$

The emergence of KRAS and PIK3CA mutations has been associated with acquired resistance to HER2 targeting in metastatic CRC with HER2 amplification. ${ }^{28}$ Preclinical PDX models have also linked RAS mutations with resistance to anti-HER2 therapy in HER2-amplified CRC. ${ }^{28}$ It is possible that the KRAS and PIK3CA mutations may have contributed partially to the resistance to HER2 targeting. However, the VAFs of KRAS and PIK3CA $(0.04 \%$ and $0.4 \%$, respectively) were extremely low compared with that of TP53 (VAF, 44.2\%), indicating that these mutations may not represent the predominant mechanism of resistance to HER2 targeting in our patient. Other studies have shown that the amplification of FGFR signaling represents another mechanism of resistance to HER2 targeting in HER2-amplified tumors. ${ }^{29,30}$ Our case report suggests the emergence of the KRAS mutation, the activation of the PI3K-AKT pathway, and FGFR2 amplification as separate and heterogeneous mechanisms of resistance to HER2 targeting.
Trastuzumab-deruxtecan is an antibody-drug conjugate that conjugates trastuzumab through a tetrapeptide-based cleavable linker to a topoisomerase I inhibitor payload. The DESTINY-CRC01 trial ${ }^{11}$ has shown a response rate to trastuzumab-deruxtecan of $45 \%$ and a median progression-free survival of 6.9 months in patients with RAS WT HER2amplified metastatic CRC. The response rate in patients with prior HER2-targeted therapy was $43.8 \%$, similar to the response rate in those who were HER2-targeting-naïve. The clinical benefit seen in our patient, albeit short, further supports the value of trastuzumab-deruxtecan beyond progression with dual HER2-targeting.

\section{Conclusions}

This is the first report of a patient with metastatic CRC with concurrent HER2 amplification and a HER2 S310F mutation who achieved an excellent clinical response to dual HER2 targeting with trastuzumab + lapatinib. He received a total of 13 cycles of trastuzumab + lapatinib, with a 10-month time to progression. Our data confirm the relevance of HER2 targeting for this specific HER2 alteration and does not extend to other HER2 tyrosine kinase alterations in metastatic CRC. Treatment with trastuzumab-deruxtecan resulted in additional clinical benefit, suggesting the relevance of this agent in tumors harboring concurrent HER2 amplification and mutation. Our case report highlights the need for HER2 assessment in all patients with CRC and provides support for further investigating HER2 targeting in HER2-mutated CRC.

Submitted December 15, 2020; revision received February 1, 2021; accepted for publication February 2, 2021.

Disclosures: Dr. Fakih has disclosed receiving honoraria from Amgen; receiving grant/research support from AstraZeneca, Amgen, and Novartis; serving as a scientific advisor for Amgen, Array, Bayer, and Pfizer; and serving on a speakers' bureau for Amgen and Guardant Health. Dr. Wang has disclosed not receiving any financial consideration from any person or organization to support the preparation, analysis, results, or discussion of this article.

Correspondence: Marwan Fakih, MD, Department of Medical Oncology and Therapeutics Research, City of Hope Comprehensive Cancer Center, 1500 East Duarte Road, Duarte, CA 91010. Email: mfakih@coh.org

\section{References}

1. Richman SD, Southward K, Chambers $P$, et al. HER2 overexpression and amplification as a potential therapeutic target in colorectal cancer: analysis of 3256 patients enrolled in the QUASAR, FOCUS and PICCOLO colorectal cancer trials. J Pathol 2016;238:562-570.

2. Bai J, Gao J, Mao Z, et al. Genetic mutations in human rectal cancers detected by targeted sequencing. J Hum Genet 2015;60:589-596.

3. Ingold Heppner B, Behrens HM, Balschun K, et al. HER2/neu testing in primary colorectal carcinoma. Br J Cancer 2014;111:1977-1984.

4. Raghav K, Loree JM, Morris JS, et al. Validation of HER2 amplification as a predictive biomarker for anti-epidermal growth factor receptor antibody therapy in metastatic colorectal cancer. JCO Precis Oncol 2019;3:1-13.

5. Bregni G, Sciallero S, Sobrero A. HER2 amplification and anti-EGFR sensitivity in advanced colorectal cancer. JAMA Oncol 2019;5:605-606.

6. Martin V, Landi L, Molinari F, et al. HER2 gene copy number status may influence clinical efficacy to anti-EGFR monoclonal antibodies in metastatic colorectal cancer patients. Br J Cancer 2013;108:668-675.

7. Bertotti A, Migliardi G, Galimi F, et al. A molecularly annotated platform of patient-derived xenografts ("xenopatients") identifies HER2 as an effective therapeutic target in cetuximab-resistant colorectal cancer. Cancer Discov 2011;1:508-523.

8. Sartore-Bianchi A, Trusolino L, Martino C, et al. Dual-targeted therapy with trastuzumab and lapatinib in treatment-refractory, KRAS codon 12/13 wild-type, HER2-positive metastatic colorectal cancer (HERACLES): 
a proof-of-concept, multicentre, open-label, phase 2 trial. Lancet Oncol 2016;17:738-746.

9. Meric-Bernstam F, Hurwitz H, Raghav KPS, et al. Pertuzumab plus trastuzumab for HER2-amplified metastatic colorectal cancer (MyPathway): an updated report from a multicentre, open-label, phase $2 a$, multiple basket study. Lancet Oncol 2019;20:518-530.

10. Benson AB III, Venook AP, Al-Hawary MM, et al. NCCN Clinical Practice Guidelines in Oncology: Colon Cancer. Version 2.2021. Accessed February 8, 2021. To view the most recent version, visit NCCN.org

11. Siena S, Bartolomeo MD, Raghav KPS, et al. A phase II, multicenter, open-label study of trastuzumab deruxtecan (T-DXd; DS-8201) in patients (pts) with HER2-expressing metastatic colorectal cancer (mCRC): DESTINY-CRC01 [abstract]. J Clin Oncol 2020;38(Suppl):Abstract 4000.

12. Ross JS, Fakih M, Ali SM, et al. Targeting HER2 in colorectal cancer: the landscape of amplification and short variant mutations in ERBB2 and ERBB3. Cancer 2018;124:1358-1373.

13. Kavuri SM, Jain N, Galimi F, et al. HER2 activating mutations are targets for colorectal cancer treatment. Cancer Discov 2015;5:832-841.

14. Ben-Baruch NE, Bose R, Kavuri SM, et al. HER2-mutated breast cancer responds to treatment with single-agent neratinib, a second-generation HER2/EGFR tyrosine kinase inhibitor. J Natl Compr Canc Netw 2015; 13:1061-1064.

15. Xu X, De Angelis $C$, Burke KA, et al. HER2 reactivation through acquisition of the HER2 L755S mutation as a mechanism of acquired resistance to HER2-targeted therapy in HER2 ${ }^{+}$breast cancer. Clin Cancer Res 2017:23:5123-5134

16. Nagano M, Kohsaka S, Ueno T, et al. High-throughput functional evaluation of variants of unknown significance in ERBB2. Clin Cancer Res 2018; 24:5112-5122.

17. Cocco $E$, Javier Carmona F, Razavi $P$, et al. Neratinib is effective in breast tumors bearing both amplification and mutation of ERBB2 (HER2). Sci Signal 2018;11:eaat9773.

18. Zuo WJ, Jiang YZ, Wang YJ, et al. Dual characteristics of novel HER2 kinase domain mutations in response to HER2-targeted therapies in human breast cancer. Clin Cancer Res 2016;22:4859-4869.

19. Pahuja KB, Nguyen TT, Jaiswal BS, et al. Actionable activating oncogenic ERBB2/HER2 transmembrane and juxtamembrane domain mutations. Cancer Cell 2018;34:792-806.e5.
20. Ou SI, Schrock AB, Bocharov EV, et al. HER2 transmembrane domain (TMD) mutations (V659/G660) that stabilize homo- and heterodimerization are rare oncogenic drivers in lung adenocarcinoma that respond to afatinib. J Thorac Oncol 2017:12:446-457.

21. Greulich H, Kaplan B, Mertins $P$, et al. Functional analysis of receptor tyrosine kinase mutations in lung cancer identifies oncogenic extracellular domain mutations of ERBB2. Proc Natl Acad Sci USA 2012;109: 14476-14481.

22. Bose R, Kavuri SM, Searleman AC, et al. Activating HER2 mutations in HER2 gene amplification negative breast cancer. Cancer Discov 2013;3:224-237.

23. Siravegna G, Sartore-Bianchi A, Nagy RJ, et al. Plasma HER2 (ERBB2) copy number predicts response to HER2-targeted therapy in metastatic colorectal cancer. Clin Cancer Res 2019;25:3046-3053.

24. Strickler JH, Zemla T, Ou FS, et al. Trastuzumab and tucatinib for the treatment of HER2 amplified metastatic colorectal cancer (mCRC): initial results from the MOUNTAINEER trial [abstract]. Ann Oncol 2019;30(Suppl 5): Abstract 527PD.

25. Nakamura Y, Okamoto W, Kato T, et al. TRIUMPH: primary efficacy of a phase II trial of trastuzumab $(T)$ and pertuzumab $(P)$ in patients (pts) with metastatic colorectal cancer (mCRC) with HER2 (ERBB2) amplification (amp) in tumour tissue or circulating tumour DNA (ctDNA): a GOZILA sub-study [abstract]. Ann Oncol 2019;30(Suppl 5):Abstract 526PD.

26. Kovacs E, Das R, Wang Q, et al. Analysis of the role of the C-terminal tai in the regulation of the epidermal growth factor receptor. Mol Cell Biol 2015;35:3083-3102

27. Kovacs E, Zorn JA, Huang Y, et al. A structural perspective on the regulation of the epidermal growth factor receptor. Annu Rev Biochem 2015;84:739-764.

28. Siravegna G, Lazzari L, Crisafulli G, et al. Radiologic and genomic evolution of individual metastases during HER2 blockade in colorectal cancer. Cancer Cell 2018;34:148-162.e7.

29. Azuma K, Tsurutani J, Sakai K, et al. Switching addictions between HER2 and FGFR2 in HER2-positive breast tumor cells: FGFR2 as a potentia target for salvage after lapatinib failure. Biochem Biophys Res Commun 2011;407:219-224.

30. Hanker AB, Garrett JT, Estrada MV, et al. HER2-overexpressing breast cancers amplify FGFR signaling upon acquisition of resistance to dual therapeutic blockade of HER2. Clin Cancer Res 2017;23:4323-4334. 